\title{
Multiplicative Cauchy functional equation and the equation of ratios on the Lorentz cone
}

\author{
by \\ JACEK WeSOŁOWSKI (Warszawa)
}

\begin{abstract}
It is proved that the solution of the multiplicative Cauchy functional equation on the Lorentz cone of dimension greater than two is a power function of the determinant. The equation is solved in full generality, i.e. no smoothness assumptions on the unknown function are imposed. Also the functional equation of ratios, of a similar nature, is solved in full generality.
\end{abstract}

1. Introduction. Consider the following problem, which looks quite natural: describe the family of positive functions of positive argument with the property that the ratio of values depends only on the ratio of arguments. Thus one seeks solutions of the following functional equation:

$$
\frac{f(x y)}{f(x(1-y))}=a(y), \quad \forall(x, y) \in(0, \infty) \times(0,1),
$$

where $f:(0, \infty) \rightarrow(0, \infty)$ and $a:(0,1) \rightarrow(0, \infty)$ are unknown functions. For obvious reasons we call this equation the functional equation of ratios.

Changing the variables $s=x y$ and $1 / t=x(1-y)$, and introducing the functions $g, b:(0, \infty) \rightarrow(0, \infty)$ by

$$
g(t)=\frac{1}{f(1 / t)}, \quad b(t)=a\left(\frac{t}{1+t}\right), \quad t \in(0, \infty),
$$

we get the Pexider version of the multiplicative Cauchy equation:

$$
f(s) g(t)=b(s t), \quad \forall(s, t) \in(0, \infty)^{2} .
$$

Thus (see for instance Aczél and Dhombres (1989)), $f(x)=H(x)$ for $x \in$ $(0, \infty)$ and $a(y)=H(y /(1-y))$ for $y \in(0,1)$, where $H$ is a generalized power function. A slightly different derivation of these solutions of equation (1) has been given recently in Ger (2004).

2000 Mathematics Subject Classification: 39B52, 39B22, 17A15, 62H05.

Key words and phrases: Cauchy equation, equation of ratios, Lorentz cone, determinant, generalized power function, Lukacs characterization. 
The aim of this paper is to study the Lorentz cone versions of equations (2) and (1), which are not so closely related in this case as they are in the univariate situation described above.

The Lorentz cone, which is a basic object of relativity theory, denoted here by $V$, is an example of a symmetric cone. Another example, more familiar to a wider mathematical audience, is the cone $\mathcal{V}_{+}$of positive definite symmetric matrices. The mutliplicative Cauchy equation on $\mathcal{V}_{+}$has been solved recently under smoothness assumptions in Bobecka and Wesołowski (2003), referred to as BW2 in the following. Recently, symmetric cones received also considerable attention in probability and mathematical statistics due to generalizations of Wishart distributions and related characterizations: see for instance Massam (1994), Casalis and Letac (1996), Massam and Neher (1997), Bobecka and Wesołowski (2002) (BW1 in what follows). To a large extent this development was enhanced by developments in the analysis on symmetric cones presented in the monograph by Faraut and Korányi (1994). In particular, in BW1, a characterization problem for the Wishart random matrix was reduced to two functional equations on $\mathcal{V}_{+}$, one of them being the equation of ratios. It should be emphasized that, in contrast to the univariate case described above, the version of the equation of ratios in $\mathcal{V}_{+}$, due to non-commutativity and non-associativity of the symmetric product $x^{1 / 2} y x^{1 / 2}$, cannot be reduced in a direct way to the Cauchy equation in $\mathcal{V}_{+}$.

In the present paper we are concerned with both these equations: the multiplicative Cauchy equation and the equation of ratios on the Lorentz cone $V$. The structure of $V$ seems to be more transparent than that of $\mathcal{V}_{+}$. Some formulas can be written explicitly for $V$, while their analogues are not available in analytical forms in $\mathcal{V}_{+}$. As an example one can consider a square root of an element in the cone (see (5) below). Consequently, the smoothness assumptions, which were essential in the solutions of functional equations on $\mathcal{V}_{+}$in BW1 and BW2, are omitted for their analogues on $V$. However, a Lorentz cone version of the second equation of BW1 still remains to be solved.

The paper is organized as follows. In Section 2 basic facts on the Lorentz cone are presented in an accessible and explicit way. In particular, for any element of $V$ we define its inverse, square root and determinant. Also a symmetric product of elements from $V$ is defined. Section 3 is devoted to the multiplicative Cauchy functional equation on $V$ and its complete solution. An interesting feature of this solution is that in the two-dimensional case it is not a generalized power function of the determinant as one may expect. A deeper reason for that is that in two dimensions the cone is not irreducible. In any dimension higher than two, the solution is, as expected, a generalized power function of the determinant. 
Section 4 is devoted to the functional equation of ratios. Here again, just as for positive definite matrices (see BW1), we do not reduce the equation to the Cauchy equation. However, there are some similarities between the methods used in the proofs. To be more precise, the first equation of BW1, which is the logarithmic version of (1) in the cone of positive definite matrices, was solved under differentiability assumptions. Here we give a solution of (1) in $V$ with no regularity assumptions on the unknown functions. Again, as for the Cauchy equation, due to reducibility of the cone, the case of dimension two implies special solutions, while in higher dimensions the solution is a generalized power function of the determinant.

2. Preliminaries on the Lorentz cone. Let $E$ be a Euclidean space with the norm $\|\cdot\|$ defined by the inner product $\langle\cdot, \cdot\rangle$. Consider the space $\mathbb{R} \times E$ with elements denoted by $\mathbf{x}=\left(x_{0}, x\right)$, where $x_{0} \in \mathbb{R}$ and $x \in E$. Then $\mathbb{R} \times E$ is Euclidean with the inner product

$$
[\mathbf{x}, \mathbf{y}]=x_{0} y_{0}+\langle x, y\rangle \text {. }
$$

Also we define a product (the Jordan product) of $\mathbf{x}$ and $\mathbf{y}$ as

$$
\mathbf{x y}=\left([\mathbf{x}, \mathbf{y}], x_{0} y+y_{0} x\right) \in \mathbb{R} \times E .
$$

If $\mathbf{x}$ and $\mathbf{y}$ are interpreted as $2 \times 2$ matrices

$$
\mathbf{x}=\left(\begin{array}{cc}
x_{0} & x \\
x & x_{0}
\end{array}\right), \quad \mathbf{y}=\left(\begin{array}{cc}
y_{0} & y \\
y & y_{0}
\end{array}\right)
$$

then the product $\mathbf{x y}$ can be viewed as the matrix product:

$$
\mathbf{x y}=\left(\begin{array}{cc}
{[\mathbf{x}, \mathbf{y}]} & x_{0} y+y_{0} x \\
x_{0} y+y_{0} x & {[\mathbf{x}, \mathbf{y}]}
\end{array}\right)
$$

Exploiting further the matrix representation, the determinant of $\mathbf{x}$ is defined as

$$
\operatorname{det}(\mathbf{x})=x_{0}^{2}-\|x\|^{2} .
$$

Also if $\operatorname{det}(\mathbf{x}) \neq 0$ then the inverse $\mathbf{x}^{-1}$ exists and equals

$$
\mathbf{x}^{-1}=\frac{1}{\operatorname{det}(\mathbf{x})}\left(\begin{array}{cc}
x_{0} & -x \\
-x & x_{0}
\end{array}\right)
$$

i.e. $\mathbf{x}^{-1}$ is the only element of $\mathbb{R} \times E$ such that $\mathbf{x}^{-1} \mathbf{x}=\mathbf{x} \mathbf{x}^{-1}=(1, \varnothing)=\mathbf{e}$, where $\varnothing$ is the zero of $E$.

However, some caution is needed while using the matrix representation since the product is commutative: $\mathbf{x y}=\mathbf{y x}$, but not associative, i.e. $(\mathbf{x y}) \mathbf{z} \neq$ $\mathbf{x}(\mathbf{y z})$.

Let $V=\left\{\mathbf{x} \in \mathbb{R} \times E: x_{0}>\|x\|\right\}$ denote the Lorentz cone in $\mathbb{R} \times E$.

Note that if $\mathbf{x}, \mathbf{y} \in V$ then $\mathbf{x y}$ may not be an element of $V$, but for $\mathbf{x} \in V$ we have $\mathbf{x} \mathbf{x} \in V$. Moreover, for any $\mathbf{x} \in V$ one can define $\mathbf{x}^{1 / 2}$ by the 
equation $\mathbf{x}^{1 / 2} \mathbf{x}^{1 / 2}=\mathbf{x}$. It is a matter of elementary calculations to see that the only solution of this equation which is an element of $V$ has the form

$$
\mathbf{x}^{1 / 2}=\frac{1}{\sqrt{2\left(x_{0}+\sqrt{\operatorname{det}(\mathbf{x})}\right)}}\left(x_{0}+\sqrt{\operatorname{det}(\mathbf{x})}, x\right) .
$$

Now we define another product which is a non-symmetric operation from $V \times V$ onto $V$. For any $\mathbf{x}, \mathbf{y} \in V$ let

$$
\mathbf{x} \circ \mathbf{y}=2 \mathbf{x}^{1 / 2}\left(\mathbf{x}^{1 / 2} \mathbf{y}\right)-\mathbf{x y} .
$$

Note that it can be rewritten as

$$
\mathbf{x} \circ \mathbf{y}=\left([\mathbf{x}, \mathbf{y}], \sqrt{\operatorname{det}(\mathbf{x})} y+\left(y_{0}+\frac{\langle x, y\rangle}{x_{0}+\sqrt{\operatorname{det}(\mathbf{x})}}\right) x\right) .
$$

Note also that (6) and $\operatorname{det}\left(\mathbf{x}^{2}\right)=(\operatorname{det}(\mathbf{x}))^{2}$ imply

$$
\mathbf{x}^{2} \circ \mathbf{y}=\left(\left[\mathbf{x}^{2}, \mathbf{y}\right], \operatorname{det}(\mathbf{x}) y+2[\mathbf{x}, \mathbf{y}] x\right) .
$$

A computation shows that the Cauchy theorem on the determinant of the product holds true for the product $\mathbf{x} \circ \mathbf{y}$, i.e.

$$
\operatorname{det}(\mathbf{x} \circ \mathbf{y})=\operatorname{det}(\mathbf{x}) \operatorname{det}(\mathbf{y}) \text {. }
$$

Observe that $\mathbf{x} \in V$ iff $x_{0}>0$ and $\operatorname{det}(\mathbf{x})>0$. Now, by (8), to prove that $\mathbf{x} \circ \mathbf{y} \in V$ it suffices to show that $[\mathbf{x}, \mathbf{y}]$ is positive; but by the Cauchy-Schwarz inequality,

$$
[\mathbf{x}, \mathbf{y}] \geq x_{0} y_{0}-|\langle x, y\rangle| \geq x_{0} y_{0}-\|x\|\|y\|>0,
$$

since $x_{0}>\|x\|$ and $y_{0}>\|y\|$.

Observe also that $\mathbf{x} \circ \mathbf{x}^{-1}=\mathbf{x}^{-1} \circ \mathbf{x}=\mathbf{e}$.

Finally, note that the product $\circ$ is neither commutative, nor associative, i.e. in general $\mathbf{x} \circ \mathbf{y} \neq \mathbf{y} \circ \mathbf{x}$ and $(\mathbf{x} \circ \mathbf{y}) \circ \mathbf{z} \neq \mathbf{x} \circ(\mathbf{y} \circ \mathbf{z})$.

The above considerations are specializations to the Lorentz cone of some basic facts from the theory of Jordan algebras and symmetric cones, which can be found in Faraut and Korányi (1994). For instance, the product xy is nothing else than the Jordan product $L(\mathbf{x}) \mathbf{y}$, and the product $\mathbf{x} \circ \mathbf{y}$ is the so called quadratic representation $P\left(\mathbf{x}^{1 / 2}\right) \mathbf{y}$.

3. The Cauchy equation. In this section we consider a Lorentz cone analogue of the multiplicative Cauchy equation which on $(0, \infty)$ (a trivial Lorentz cone with $E=\{\varnothing\}$ ) has the form

$$
f(x y)=f(x) f(y) \quad \text { for any } x, y>0,
$$

where $f:(0, \infty) \rightarrow(0, \infty)$. It is well known that the set of solutions of $(9)$ is the class of so called generalized power functions, i.e. $f=H$, where $H$ is a generalized power function. In this sense the equation (9) can be treated as the definition of the class of generalized power functions. Under very weak 
conditions any generalized power function $H$ has the form $H(x)=x^{a}$ for some real number $a$.

There are numerous extensions of the equation (9) in the literature. Many of them deal with its versions on abstract algebraic-topological structures. One of the most recent, given in BW2, is concerned with (9) on the cone $\mathcal{V}_{+}$of positive definite real symmetric matrices of a fixed dimension. The equation (9) then reads

$$
f\left(x^{1 / 2} y x^{1 / 2}\right)=f(x) f(y)
$$

for any $x, y \in \mathcal{V}_{+}$. It is shown in that paper that if (10) holds then there exists a real number $\lambda$ such that $f(x)=[\operatorname{det}(x)]^{\lambda}$ for any $x \in \mathcal{V}_{+}$. A drawback of this result is that it was obtained under an additional assumption of differentiability of $f$.

Note that the cone $\mathcal{V}_{+}$, just as the Lorentz cone $V$, is an example of a symmetric cone. The main result of this note is a solution of a version of (10) in the Lorentz cone. The solution is given in full generality, i.e. without any smoothness assumption on the unknown function.

TheOREM 1. Let $V$ be a Lorentz cone in $\mathbb{R} \times$ E. Let $f: V \rightarrow(0, \infty)$ satisfy the equation

$$
f(\mathbf{x} \circ \mathbf{y})=f(\mathbf{x}) f(\mathbf{y})
$$

for any $\mathbf{x}, \mathbf{y} \in V$, where the product $\circ$ is defined by (6). Then either the dimension of $E$ is greater than 1 and

$$
f(\mathbf{x})=H(\operatorname{det}(\mathbf{x})) \quad \text { for any } \mathbf{x} \in V,
$$

where $H$ is a generalized power function and the determinant det is defined by (3), or $E$ is univariate, i.e. $E=\left\{x_{1} e: x_{1} \in \mathbb{R}\right\}$, and

$$
f(\mathbf{x})=H_{1}\left(x_{0}+x_{1}\right) H_{2}\left(x_{0}-x_{1}\right) \quad \text { for any } \mathbf{x}=\left(x_{0}, x_{1} e\right),
$$

where $H_{1}$ and $H_{2}$ are generalized power functions.

Proof. Take $\mathbf{x}=(a, \varnothing)$ and $\mathbf{y}=(b, \varnothing)$ in (11). Then

$$
f(a b, \varnothing)=f(a, \varnothing) f(b, \varnothing)
$$

for any $a, b \in(0, \infty)$. Consequently, the function $f(\cdot, \varnothing)$ defined on $(0, \infty)$ is a power function, i.e. $f(a, \varnothing)=H(a)$ for any $a>0$, where $H$ is a power function.

Define a function $G$ on $(0, \infty) \times E$ by

$$
G(\operatorname{det}(\mathbf{x}), x)=f(\mathbf{x})
$$

for any $\mathbf{x}=\left(x_{0}, x\right) \in V$. Consider (11) for $\mathbf{x}^{2}=\mathbf{x} \mathbf{x}$ instead of $\mathbf{x}$. Note that $\operatorname{det}\left(\mathbf{x}^{2}\right)=[\operatorname{det}(\mathbf{x})]^{2}$. Consequently, by (8) and (7) the equation (11) with this change of variable can be written as 


$$
\begin{aligned}
G\left([\operatorname{det}(\mathbf{x})]^{2} \operatorname{det}(\mathbf{y}), \operatorname{det}(\mathbf{x}) y+2[\mathbf{x}, \mathbf{y}] x\right) \\
=G\left([\operatorname{det}(\mathbf{x})]^{2}, 2 x_{0} x\right) G(\operatorname{det}(\mathbf{y}), y) .
\end{aligned}
$$

Insert into (13) the variables $\mathbf{x}$ and $\mathbf{y}$ such that $\operatorname{det}(\mathbf{x})=\operatorname{det}(\mathbf{y})=1$. Then for a function $F$ on $E$ defined by $F(x)=G(1, x)$ for any $x \in E$, we have

$$
F\left(y+2\left[\sqrt{\left(1+\|y\|^{2}\right)\left(1+\|x\|^{2}\right)}+\langle x, y\rangle\right] x\right)=F\left(2 \sqrt{1+\|x\|^{2}} x\right) F(y)
$$
for all $x, y \in E$.

In the above equation, insert $2 x \sqrt{1+\|x\|^{2}}=\alpha u$ and $y=\beta u$ where $\alpha$ and $\beta$ are arbitrary real numbers and $u \in E$ has length 1 . Then, after some straightforward transformations, we get

$$
F\left(\left[\alpha \sqrt{1+\beta^{2}}+\beta \sqrt{1+\alpha^{2}}\right] u\right)=F(\alpha u) F(\beta u) .
$$

For any $u \in E$ with $\|u\|=1$ define $g_{u}: \mathbb{R} \rightarrow \mathbb{R}$ by $g_{u}(\alpha)=F(\alpha u), \alpha \in \mathbb{R}$. Then, for any $\alpha, \beta \in \mathbb{R}$ the above equation can be written as

$$
g_{u}\left(\alpha \sqrt{1+\beta^{2}}+\beta \sqrt{1+\alpha^{2}}\right)=g_{u}(\alpha) g_{u}(\beta) .
$$

Note that for the real function $h$ defined by $h(x)=x+\sqrt{1+x^{2}}, x \in \mathbb{R}$, we have

$$
h\left(\alpha \sqrt{1+\beta^{2}}+\beta \sqrt{1+\alpha^{2}}\right)=h(\alpha) h(\beta)
$$

for any real $\alpha$ and $\beta$. The function $h$ is a bijection of $\mathbb{R}$ onto $(0, \infty)$. Now, we define $T_{u}=g_{u} \circ h^{-1}$. Then (15) can be rewritten as

$$
T_{u}(h(\alpha) h(\beta))=T_{u}(h(\alpha)) T_{u}(h(\beta)), \quad \alpha, \beta \in \mathbb{R} .
$$

It follows that $T_{u}$ is a generalized power function $H_{u}$ and $g_{u}=H_{u} \circ h$.

Now consider the case of univariate $E$. Then

$$
F(x)=F\left(x_{1} e\right)=g_{e}\left(x_{1}\right)=H_{e}\left(x_{1}+\sqrt{1+x_{1}^{2}}\right), \quad x_{1} \in \mathbb{R} .
$$

If the dimension of $E$ is greater than 1, then we can choose orthogonal $x, y$ in (14). For such $x$ and $y$ that equation reads

$$
F\left(y+2 \sqrt{\left(1+\|y\|^{2}\right)\left(1+\|x\|^{2}\right)} x\right)=F\left(2 \sqrt{1+\|x\|^{2}} x\right) F(y) .
$$

Thus for any $y, z \in E$ such that $\langle y, z\rangle=0$ we have

$$
F(y+z)=F\left(z / \sqrt{1+\|y\|^{2}}\right) F(y) .
$$

This follows from the fact that the last equation can be reduced to the previous one by defining $x$ through $z=2 \sqrt{\left(1+\|x\|^{2}\right)\left(1+\|y\|^{2}\right)} x$. It can be checked by simple computation, involving solution of a quadratic equation, that $x$ is correctly defined.

Now, by symmetry, we get

$$
F\left(z / \sqrt{1+\|y\|^{2}}\right) F(y)=F\left(y / \sqrt{1+\|z\|^{2}}\right) F(z)
$$

if only $\langle y, z\rangle=0$. Since $F$ is positive we can rewrite the above equation as 


$$
\frac{F\left(z / \sqrt{1+\|y\|^{2}}\right)}{F(z)}=\frac{F\left(y / \sqrt{1+\|z\|^{2}}\right)}{F(y)}
$$

for $\langle y, z\rangle=0$. Insert into (18) new variables $z=\alpha u$ and $y=\beta v$, where $\alpha, \beta$ are any real numbers and $u, v$ are orthogonal unit vectors. Then, upon using the properties of the generalized power function, we arrive at

$$
H_{u}\left(\frac{\alpha+\sqrt{1+\alpha^{2}+\beta^{2}}}{\sqrt{1+\beta^{2}}\left(\alpha+\sqrt{1+\alpha^{2}}\right)}\right)=H_{v}\left(\frac{\beta+\sqrt{1+\alpha^{2}+\beta^{2}}}{\sqrt{1+\alpha^{2}}\left(\beta+\sqrt{1+\beta^{2}}\right)}\right)
$$

for any $\alpha, \beta \in \mathbb{R}$. Now for $\alpha=\beta$ one gets

$$
H_{u}\left(\frac{\alpha+\sqrt{1+2 \alpha^{2}}}{\sqrt{1+\alpha^{2}}\left(\alpha+\sqrt{1+\alpha^{2}}\right)}\right)=H_{v}\left(\frac{\alpha+\sqrt{1+2 \alpha^{2}}}{\sqrt{1+\alpha^{2}}\left(\alpha+\sqrt{1+\alpha^{2}}\right)}\right)
$$

for any real $\alpha$. Since the argument as a function of $\alpha$ is a surjection from $\mathbb{R}$ onto $(0, \infty)$ we conclude that $H_{u}=H_{v}$ for any orthogonal unit vectors $u$ and $v$. Consequently, we can replace $H_{v}$ on the right hand side of (19) by $H_{u}$. Taking then $\beta=-\alpha$ we obtain

$$
H_{u}\left(\frac{\alpha+\sqrt{1+2 \alpha^{2}}}{\alpha+\sqrt{1+\alpha^{2}}}\right)=H_{u}\left(\frac{-\alpha+\sqrt{1+2 \alpha^{2}}}{-\alpha+\sqrt{1+\alpha^{2}}}\right)
$$

for any real $\alpha$. Thus, as $H_{u}$ is a generalized power function we have

$$
H_{u}\left(\frac{\left(\sqrt{1+2 \alpha^{2}}-\alpha\right)\left(\sqrt{1+\alpha^{2}}+\alpha\right)}{\left(\sqrt{1+2 \alpha^{2}}+\alpha\right)\left(\sqrt{1+\alpha^{2}}-\alpha\right)}\right)=1 \text {. }
$$

Note that the argument of $H_{u}$ in (20) is a continuous positive function on $\mathbb{R}$, say $r$, evaluated at $\alpha \in \mathbb{R}$. Moreover $\lim _{\alpha \rightarrow-\infty} r(\alpha)=0$ and $\lim _{\alpha \rightarrow \infty} r(\alpha)=\infty$. Thus $r: \mathbb{R} \rightarrow(0, \infty)$ is surjective. Consequently, $H_{u} \equiv 1$ for any $u \in E$ such that $\|u\|=1$.

Finally, we conclude that $g_{u} \equiv 1$, which implies that $1 \equiv F(\cdot)=G(1, \cdot)$.

Insert now into (13) the variables

$$
\mathbf{x}=\left(a^{1 / 4}, \varnothing\right) \quad \text { and } \quad \mathbf{y}=\left(\sqrt{1+\|y\|^{2} / a}, y / \sqrt{a}\right)
$$

for any $a>0$ and $y \in E$. Note that $\operatorname{det}(\mathbf{y})=1$. Then $G$ is expressed in terms of $G(\cdot, \varnothing)=f(\cdot, \varnothing)=H$ and $G(1, \cdot)=F$ as

$$
G(a, y)=H(a) F(y / \sqrt{a}) .
$$

Finally, if the dimension of $E$ is greater than one, since $F \equiv 1$, it suffices to refer to the definition of $G$ given in (12). In the case of univariate $E$ one has to refer to (16). Then (12) implies

$$
\begin{aligned}
f\left(x_{0}, x_{1}\right) & =H\left(x_{0}^{2}-x_{1}^{2}\right) H_{e}\left(\frac{x_{1}}{\sqrt{x_{0}^{2}-x_{1}^{2}}}+\sqrt{1+\frac{x_{1}^{2}}{x_{0}^{2}-x_{1}^{2}}}\right) \\
& =H_{1}\left(x_{0}+x_{1}\right) H_{2}\left(x_{0}-x_{1}\right)
\end{aligned}
$$

for some generalized power functions $H_{1}$ and $H_{2}$. 
4. The equation of ratios. In BW1 the authors proved a characterization of the Wishart distribution on the cone of positive definite symmetric matrices, which was a new version of the celebrated Lukacs characterization of the gamma distribution on $(0, \infty)$. The main novelty of that result was that no invariance of the distribution of the "quotient" $(X+Y)^{-1 / 2} X(X+$ $Y)^{-1 / 2}$ was imposed. The invariance property was rather crucial in earlier results of this kind, like those of Olkin and Rubin (1962), Casalis and Letac (1996) and Letac and Massam (1998). However, in BW1 technical smoothness assumptions were imposed on the densities of the random matrices considered. Those assumptions played an important role in solving two functional equations. As mentioned earlier, the first of the equations (see Theorem 2 in BW1) is a version of the functional equation of ratios (1) in $\mathcal{V}_{+}$:

$$
a(y)=\frac{f\left(x^{1 / 2} y x^{1 / 2}\right)}{f\left(x^{1 / 2}(e-y) x^{1 / 2}\right)}
$$

for any $x \in \mathcal{V}_{+}$and $y \in \mathcal{D}=\left\{z \in \mathcal{V}_{+}: e-z \in \mathcal{V}_{+}\right\}$, where $e$ is the identity matrix, and $a: \mathcal{D} \rightarrow(0, \infty), f: \mathcal{V}_{+} \rightarrow(0, \infty)$. The equation (21) was solved under the assumption that $f$ is differentiable.

The second equation (see Theorem 3 in BW1) was more involved and a more restrictive smoothness assumption on one of the unknown functions was imposed.

It is worth mentioning that the approach to Lukacs-type characterization developed in BW1 has been recently used to obtain its analogue on homogeneous cones in Boutouria (2005). In that paper again smoothness assumptions were imposed on functions which appeared in two functional equations, analogous to the equations from BW1. Thus the version of the functional equation of ratios for homogeneous cones was solved there under a differentiability condition. Note that any symmetric cone, hence also the Lorentz cone, is homogeneous. Thus the Lukacs-type characterization of the Wishart distribution on the Lorentz cone, under smoothness assumptions, follows from the result of Boutouria (2005). Unfortunately, we are unable to solve the Lorentz cone version of the second equation of BW1 in full generality. Consequently, no improvement of the Wishart characterization is available at present.

Our goal in this section is to solve the Lorentz cone version of the equation of ratios (1) (or (21)) without any smoothness conditions. This seems of interest not only in its own right, but also as a first step towards a possible improvement of the Lukacs-type result for the Lorentz cone.

TheOREM 2. Let $V$ be a Lorentz cone in $\mathbb{R} \times E$. Set $D=\{\mathbf{x} \in V$ : $\mathbf{e}-\mathbf{x} \in V\}$. Let $a: D \rightarrow(0, \infty)$ and $f: V \rightarrow(0, \infty)$ satisfy 


$$
a(\mathbf{y})=\frac{f(\mathbf{x} \circ \mathbf{y})}{f(\mathbf{x} \circ(\mathbf{e}-\mathbf{y}))}, \quad \forall \mathbf{y} \in D, \forall \mathbf{x} \in V .
$$

If the dimension of $E$ is greater than one, then there exists a positive constant $\lambda$ such that

$$
f(\mathbf{x})=\lambda H(\operatorname{det}(\mathbf{x})), \quad \mathbf{x} \in V, \quad a(\mathbf{y})=H\left(\operatorname{det}\left(\mathbf{y}(\mathbf{e}-\mathbf{y})^{-1}\right)\right), \quad \mathbf{y} \in D,
$$

where $H$ is a generalized power function.

In the case of univariate $E=\left\{x_{1} e: x_{1} \in \mathbb{R}\right\}$ there exists a positive constant $\lambda$ such that for any $\mathbf{x}=\left(x_{0}, x_{1} e\right) \in V$ and any $\mathbf{y}=\left(y_{0}, y_{1} e\right) \in D$,

$$
\begin{aligned}
f\left(x_{0}, x_{1} e\right) & =\lambda H_{1}\left(x_{0}-x_{1}\right) H_{2}\left(x_{0}+x_{1}\right), \\
a\left(y_{0}, y_{1} e\right) & =\frac{H_{1}\left(y_{0}-y_{1}\right) H_{2}\left(y_{0}+y_{1}\right)}{H_{1}\left(1-y_{0}+y_{1}\right) H_{2}\left(1-y_{0}-y_{1}\right)},
\end{aligned}
$$

where $H_{1}$ and $H_{2}$ are generalized power functions.

Proof. Take $\mathbf{x}=\mathbf{e}$ in (22). Then for any $\mathbf{x} \in D$,

$$
a(\mathbf{y})=\frac{f(\mathbf{y})}{f(\mathbf{e}-\mathbf{y})} .
$$

Consequently, for any $\mathbf{x} \in V$ and $\mathbf{y} \in D$,

$$
\frac{f(\mathbf{y})}{f(\mathbf{e}-\mathbf{y})}=\frac{f(\mathbf{x} \circ \mathbf{y})}{f(\mathbf{x} \circ(\mathbf{e}-\mathbf{y}))} .
$$

Plugging $\mathbf{y}=\mathbf{x}^{-1}$ into the above equation one gets

$$
\frac{f(\mathbf{y})}{f(\mathbf{e}-\mathbf{y})}=\frac{f(\mathbf{e})}{f\left(\mathbf{y}^{-1}-\mathbf{e}\right)}, \quad \mathbf{y} \in D
$$

Define a new function $i$ on $V$ by $i(\mathbf{x})=f(\mathbf{x}) / f(\mathbf{e}), \mathbf{x} \in V$. Then combining the above two equations we have

$$
i(\mathbf{x} \circ \mathbf{y}) i\left(\mathbf{y}^{-1}-\mathbf{e}\right)=i(\mathbf{x} \circ(\mathbf{e}-\mathbf{y}))
$$

for any $\mathbf{x} \in V$ and $\mathbf{y} \in D$. Define now $G:(0, \infty) \times E \rightarrow \mathbb{R}$ by

$$
G(\operatorname{det}(\mathbf{x}), x)=i(\mathbf{x}), \quad \mathbf{x}=\left(x_{0}, x\right) \in V .
$$

Note that, by (8), for any $\mathbf{y} \in D$,

$$
\operatorname{det}\left(\mathbf{y}^{-1}-\mathbf{e}\right)=\frac{\operatorname{det}(\mathbf{e}-\mathbf{y})}{\operatorname{det}(\mathbf{y})} .
$$

Then, for $\mathbf{x}$ changed to $\mathbf{x}^{2}$ the equation (24) can be written as (see (7))

$$
\begin{array}{r}
G\left([\operatorname{det}(\mathbf{x})]^{2} \operatorname{det}(\mathbf{y}), \operatorname{det}(\mathbf{x}) y+2[\mathbf{x}, \mathbf{y}] x\right) G\left(\frac{\operatorname{det}(\mathbf{e}-\mathbf{y})}{\operatorname{det}(\mathbf{y})},-\frac{y}{\operatorname{det}(\mathbf{y})}\right) \\
=G\left([\operatorname{det}(\mathbf{x})]^{2} \operatorname{det}(\mathbf{e}-\mathbf{y}),-\operatorname{det}(\mathbf{x}) y+2[\mathbf{x}, \mathbf{e}-\mathbf{y}] x\right)
\end{array}
$$

for any $\mathbf{x} \in V$ and $\mathbf{y} \in D$. Now we insert into (26) $\mathbf{y}=(a, \varnothing)$ and $\mathbf{x}$ such that $\operatorname{det}(\mathbf{x})=1 /(1-a)$ for some $a \in(0,1)$ to get 


$$
G\left(\left(\frac{a}{1-a}\right)^{2}, 2 a x_{0} x\right) G\left(\left(\frac{1-a}{a}\right)^{2}, \varnothing\right)=G\left(1,2(1-a) x_{0} x\right)
$$

for any $a \in(0,1)$ and $x \in E$ with $x_{0}^{2}-\|x\|^{2}=(1-a)^{-1}$. Define $z=2 a x_{0} x$ $\in E$ and $\alpha=\left(\frac{a}{1-a}\right)^{2}>0$. Then

$$
G(\alpha, z)=\frac{G(1, z / \sqrt{\alpha})}{G(1 / \alpha, \varnothing)}
$$

for any $\alpha \in(0, \infty)$ and $z \in E$.

Using the above identity we rewrite (26) as

$$
\begin{array}{r}
\frac{G\left(1, \frac{\operatorname{det}(\mathbf{x}) y+2[\mathbf{x}, \mathbf{y}] x}{\operatorname{det}(\mathbf{x}) \sqrt{\operatorname{det}(\mathbf{y})})} \cdot \frac{G\left(1,-\frac{y}{\sqrt{\operatorname{det}(\mathbf{y}) \operatorname{det}(\mathbf{e}-\mathbf{y})}}\right)}{G\left(\frac{1}{(\operatorname{det}(\mathbf{x}))^{2} \operatorname{det}(\mathbf{y})}, \varnothing\right)}\right.}{} \\
=\frac{G\left(1, \frac{-\operatorname{det}(\mathbf{x}) y+2[\mathbf{x}, \mathbf{e}-\mathbf{y}] x}{\operatorname{det}(\mathbf{e}-\mathbf{y})}, \varnothing\right)}{G\left(\frac{\mathbf{d e t}) \sqrt{\operatorname{det}(\mathbf{e}-\mathbf{y})}}{(\operatorname{det}(\mathbf{x}))^{2} \operatorname{det}(\mathbf{e}-\mathbf{y})}, \varnothing\right)}
\end{array}
$$

for any $\mathbf{x} \in V$ and $\mathbf{y} \in D$. Inserting $\mathbf{y}=(a, \varnothing)$ and $\mathbf{x}=(b, \varnothing)$ we get

$$
G\left(\frac{1}{b^{4} a^{2}}, \varnothing\right) G\left(\frac{a^{2}}{(1-a)^{2}}, \varnothing\right)=G\left(\frac{1}{b^{4}(1-a)^{2}}, \varnothing\right)
$$

for any $a \in(0,1)$ and $b \in(0, \infty)$. Now taking $t=a^{2} /(1-a)^{2}$ and $u=$ $1 /\left(b^{4} a^{2}\right)$ we get

$$
G(u t, \varnothing)=G(u, \varnothing) G(t, \varnothing)
$$

for any $t, u \in(0, \infty)$. Thus $G(\cdot, \varnothing)=H(\cdot)$, where $H$ is a generalized power function.

Using (29) in (28) we can cancel denominators and arrive at

$$
\begin{aligned}
G\left(1, \frac{\operatorname{det}(\mathbf{x}) y+2[\mathbf{x}, \mathbf{y}] x}{\operatorname{det}(\mathbf{x}) \sqrt{\operatorname{det}(\mathbf{y})}}\right) \cdot G(1, & \left.-\frac{y}{\sqrt{\operatorname{det}(\mathbf{y}) \operatorname{det}(\mathbf{e}-\mathbf{y})}}\right) \\
& =G\left(1, \frac{-\operatorname{det}(\mathbf{x}) y+2[\mathbf{x}, \mathbf{e}-\mathbf{y}] x}{\operatorname{det}(\mathbf{x}) \sqrt{\operatorname{det}(\mathbf{e}-\mathbf{y})}}\right)
\end{aligned}
$$

for any $\mathbf{x} \in D$ and $\mathbf{y} \in V$.

Taking $y_{0}=0.5$ we get $\operatorname{det}(\mathbf{y})=\operatorname{det}(\mathbf{e}-\mathbf{y})$ and the above equation can be written as

$$
\begin{aligned}
F\left(\frac{\operatorname{det}(\mathbf{x}) y+2[\mathbf{x}, \mathbf{y}] x}{\operatorname{det}(\mathbf{x}) \sqrt{\operatorname{det}(\mathbf{y})}) \cdot F\left(-\frac{y}{\operatorname{det}(\mathbf{y})}\right)}\right. \\
=F\left(\frac{-\operatorname{det}(\mathbf{x}) y+2[\mathbf{x}, \mathbf{e}-\mathbf{y}] x}{\operatorname{det}(\mathbf{x}) \sqrt{\operatorname{det}(\mathbf{y})}}\right)
\end{aligned}
$$

for $F(\cdot)=G(1, \cdot)$. 
Taking in the above equation $x_{0}$ such that $\operatorname{det}(\mathbf{x})=(\operatorname{det}(\mathbf{y}))^{-1 / 2}$, i.e.

$$
x_{0}=\sqrt{\frac{1}{\sqrt{1 / 4-\|y\|^{2}}+\|x\|^{2}},}
$$

we get

$$
\begin{aligned}
& F\left(x \sqrt{\frac{1}{\sqrt{1 / 4-\|y\|^{2}}}+\|x\|^{2}}+\frac{y}{\sqrt{1 / 4-\|y\|^{2}}}\right.+2\langle x, y\rangle x) \\
& \times F\left(-\frac{y}{1 / 4-\|y\|^{2}}\right) \\
&=F\left(x \sqrt{\frac{1}{\sqrt{1 / 4-\|y\|^{2}}}+\|x\|^{2}}-\frac{y}{\sqrt{1 / 4-\|y\|^{2}}}-2\langle x, y\rangle x\right) .
\end{aligned}
$$

We plug into this equation $y=\alpha u,|\alpha|<1 / 2$, and $x=\beta u, \beta \in \mathbb{R}$, where $u \in E$ is such that $\|u\|=1$. Thus, for the function $g_{u}$ defined on $\mathbb{R}$ by $g_{u}(\alpha)=F(\alpha u)$, we get the equation

$$
\begin{array}{r}
g_{u}\left(\beta \sqrt{\frac{1}{\sqrt{1 / 4-\alpha^{2}}}+\beta^{2}}+\frac{\alpha}{\sqrt{1 / 4-\alpha^{2}}}+2 \alpha^{2} \beta\right) g_{u}\left(-\frac{\alpha}{1 / 4-\alpha^{2}}\right) \\
=g_{u}\left(\beta \sqrt{\frac{1}{\sqrt{1 / 4-\alpha^{2}}}+\beta^{2}}-\frac{\alpha}{\sqrt{1 / 4-\alpha^{2}}}-2 \alpha^{2} \beta\right) .
\end{array}
$$

Changing the variable

$$
\gamma=\beta \sqrt{\frac{1}{\sqrt{1 / 4-\alpha^{2}}}+\beta^{2}}-\frac{\alpha}{\sqrt{1 / 4-\alpha^{2}}}-2 \alpha^{2} \beta,
$$

after some tedious computation we get

$$
g_{u}\left(\frac{\gamma / 4+\alpha^{2} \gamma+\alpha \sqrt{1+\gamma^{2}}}{1 / 4-\alpha^{2}}\right) g_{u}\left(-\frac{\alpha}{1 / 4-\alpha^{2}}\right)=g_{u}(\gamma)
$$

for any $\gamma \in \mathbb{R}$ and $\alpha \in \mathbb{R}$ such that $|\alpha|<1 / 2$. Setting $\gamma=\alpha=0$ we get $g_{u}(0)=1$, since $g_{u}(0)=0$ is forbidden by the assumption. Now we set $\gamma=0$ in $(32)$ and define $v(\alpha)=\alpha /\left(1 / 4-\alpha^{2}\right)$. Note that $v:(-1 / 2,1 / 2) \rightarrow \mathbb{R}$ is surjective. Hence $g_{u}(v) g_{u}(-v)=1$ for any real $v$. Introducing now

$$
\delta=\frac{\alpha}{1 / 4-\alpha^{2}},
$$

after some easy transformations we get

$$
g_{u}\left(\gamma \sqrt{1+\delta^{2}}+\delta \sqrt{1+\gamma^{2}}\right)=g_{u}(\gamma) g_{u}(\delta)
$$

for any real $\gamma$ and $\delta$, i.e. the same equation as in the earlier proof (see (15)). 
Thus, repeating the reasoning used in the proof of Theorem 1, we obtain

$$
g_{u}(\alpha)=H_{u}\left(\alpha+\sqrt{1+\alpha^{2}}\right), \quad \alpha \in \mathbb{R} .
$$

Consequently, for univariate $E=\left\{x_{1} e: x_{1} \in \mathbb{R}\right\}$ we have

$$
F(x)=F\left(x_{1} e\right)=g_{e}\left(x_{1}\right)=H_{e}\left(x_{1}+\sqrt{1+x_{1}^{2}}\right), \quad x_{1} \in \mathbb{R} .
$$

If the dimension of $E$ is greater than one, we take $x, y \in E$ orthogonal in (31). Since $F(x) F(-x)=1$, we then have

$$
\begin{aligned}
& F\left(\frac{y}{\sqrt{1 / 4-\|y\|^{2}}}+x \sqrt{\frac{1}{\sqrt{1 / 4-\|y\|^{2}}}+\|x\|^{2}}\right) \\
& \quad \times F\left(\frac{y}{\sqrt{1 / 4-\|y\|^{2}}}-x \sqrt{\frac{1}{\sqrt{1 / 4-\|y\|^{2}}}+\|x\|^{2}}\right)=F\left(\frac{y}{1 / 4-\|y\|^{2}}\right) .
\end{aligned}
$$

Changing variables into

$$
\alpha u=\frac{y}{\sqrt{1 / 4-\|y\|^{2}}}, \quad \beta v=x \sqrt{\frac{1}{\sqrt{1 / 4-\|y\|^{2}}}+\|x\|^{2}},
$$

where $\alpha, \beta$ are real numbers and $u, v$ are orthogonal unit vectors, we obtain

$$
F(\alpha u+\beta v) F(\alpha u-\beta v)=F(\alpha u)
$$

for any $\alpha, \beta \in \mathbb{R}$ and any pair of orthogonal unit vectors $u$ and $v$. The right hand side can be expressed in terms of $g_{u}$ and consequently in terms of a generalized power function $H_{u}$ function evaluated at $\alpha+\sqrt{1+\alpha^{2}}$. But the left hand side is a product of two generalized power functions: $H_{(\alpha u+\beta v) / \sqrt{\alpha^{2}+\beta^{2}}}$ and $H_{(\alpha u-\beta v) / \sqrt{\alpha^{2}+\beta^{2}}}$ evaluated at the same point $\sqrt{\alpha^{2}+\beta^{2}}$. This product is again a generalized power function, denoted $H_{(\alpha, \beta)}$. Consequently,

$$
H_{(\alpha, \beta)}\left(\sqrt{\alpha^{2}+\beta^{2}}\right)=H_{u}\left(\alpha+\sqrt{1+\alpha^{2}}\right)
$$

for any real $\alpha$ and $\beta$. If $\alpha, \beta \in \mathbb{R}$, are such that $\alpha^{2}+\beta^{2}=1$, then the left hand side of the above equation is 1 , and thus for $\alpha$ such that $|\alpha|<1$ we get

$$
H_{u}\left(\alpha+\sqrt{1+\alpha^{2}}\right)=1 \text {. }
$$

Rising the argument to a suitable power one can get any positive number. Consequently, $H_{u} \equiv 1$.

Finally, if the dimension of $E$ is greater than one we use (33), the definition of $G$ in terms of $g_{u}$ and the representation (27) to recover $i$ through (25). Then the $f$ of (22) in Theorem 2 is obtained by its definition in terms of $i$. If $E$ is univariate the result follows by (34), (27) and (25).

Acknowledgements. I am grateful to Roman Ger for sharing with me his solution of the univariate equation of ratios. Thanks are also due to Marek Bożejko, Włodzimierz Bryc and Gérard Letac for inspiring discussions, and 
to the referee, whose remarks led to improvements in the presentation of the material.

\section{References}

J. Aczél and J. Dhombres (1989), Functional Equations in Several Variables, Cambridge Univ. Press, Cambridge.

K. Bobecka and J. Wesołowski (2002), The Lukacs-Olkin-Rubin theorem without invariance of the "quotient", Studia Math. 152, 147-160.

K. Bobecka and J. Wesołowski (2003), Multiplicative Cauchy functional equation in the cone of positive definite symmetric matrices, Ann. Polon. Math. 82, 1-7.

I. Boutouria, Characterization of the Wishart distributions on homogeneous cones, C. R. Math. Acad. Sci. Paris 341 (2005), 43-48.

M. Casalis and G. Letac (1996), The Lukacs-Olkin-Rubin characterization of Wishart distributions on symmetric cones, Ann. Statist. 24, 763-786.

J. Faraut and A. Korányi (1994), Analysis on Symmetric Cones, Clarendon Press, Oxford.

R. Ger (2004), unpublished note, 2 pp.

G. Letac and H. Massam (1998), Quadratic and inverse regressions for Wishart distributions, Ann. Statist. 26, 573-595.

H. Massam (1994), An exact decomposition theorem and a unified view of some related distributions for a class of exponential transformation models on symmetric cones, ibid. 22, 369-394.

H. Massam and E. Neher (1997), On transformations and determinants of Wishart variables on symmetric cones, J. Theoret. Probab. 10, 867-902.

I. Olkin and H. Rubin (1962), A characterization of the Wishart distribution, Ann. Math. Statist. 33, 1272-1280.

Wydział Matematyki i Nauk Informacyjnych

Politechnika Warszawska

00-661 Warszawa, Poland

E-mail: wesolo@mini.pw.edu.pl

Received November 12, 2005

Revised version January 30, 2007 